1988-01-01

\title{
Comparison of Effective Medium Procedures for Optical Modeling of Laminar Structures
}

\author{
David D. Allred \\ allred@byu.edu \\ Robert F. Edgerton
}

Follow this and additional works at: https://scholarsarchive.byu.edu/facpub

Part of the Astrophysics and Astronomy Commons, and the Physics Commons

\section{Original Publication Citation}

R.F. Edgerton and D.D. Allred, "Comparison of Effective Medium Procedures for Optical Modeling of Laminar Structures," Modeling of Optical Thin Film, M. Jacobson, Editor, Proceedings of SPIE 821, 167173 (1988).

\section{BYU ScholarsArchive Citation}

Allred, David D. and Edgerton, Robert F., "Comparison of Effective Medium Procedures for Optical Modeling of Laminar Structures" (1988). Faculty Publications. 1201.

https://scholarsarchive.byu.edu/facpub/1201

This Peer-Reviewed Article is brought to you for free and open access by BYU ScholarsArchive. It has been accepted for inclusion in Faculty Publications by an authorized administrator of BYU ScholarsArchive. For more information, please contact ellen_amatangelo@byu.edu. 
Comparison of Effective Medium Procedures for Optical Modeling of Laminar Structures

Robert F. Edgerton

Ovonic Imaging Systems

1896 Barrett St., Troy, MI 48084

and

David D. Allred

Department of Physics and Astronomy

and Center for X-Ray Optics

Brigham Young University, Provo, UT 84604

\begin{abstract}
This study addresses the question, "How can the optical properties of matter in ultrathin amorphous nonmetallic films in multilayers best be determined from reflectance $(R)$ and transmission $(T)$ measurements." A blue shift in the band gap of plasma CVD a-Si:H/a-SiN $\mathbf{x}: \mathrm{H}$ multilayers was reported several years ago. It was suggested that the shift in the band gap, $E_{y}$, relative to bulk a-Si:H as given by the Tauç plot was due to quantum confinement effects. The purpose of this study is to evaluate the usefulness of various effective media theories $(E M T)$ for determining the optical constants of materials in a multilayer and to explore to what extent a shift in band gap to higher energy may be an artifact of the method of optical analysis.
\end{abstract}

Incoherent approaches are the most common methods of determining band gap from $R$ and $T$. These do not require iteration to obtain optical constants from the optical data. The band gap determined by such methods was, however, generally $8 \%$ higher than the actual band gap when a suitable hypothetical case was investigated. Coherent effective media theory provides a noteworthy alternative to both incoherent $E M T$ and fully coherent multilayer modeling, (which is accurate but is excessively complicated and poorly convergent). The accuracy of the band gap is at the limit, $2-3 \%$, of what can be expected for graphical methods. A previously unappreciated source of optical artifacts was also identified. Dispersion, which is commonly ignored when $E_{\theta}$ is determined graphically, is shown to distort, in certain cases, the anticipated straight line behavior of the $\sqrt{a E}$ vs. $E$ plot.

\section{Introduction}

The properties of materials in ultrathin films frequently differ from the same materials in thicker films and in bulk form. Optical properties and electrical transport of metallic and semiconductor films provide many examples of this. ${ }^{2}$ The changes have been related to film discontinuity, for example agglomeration, surface oxides, inclusion of residual gas, voids, and microstructural or surface morphological differences. ${ }^{3-6}$

The purpose of this study is to investigate the suitability of various methods of determining optical constants of ultrathin $(0.5 \mathrm{~nm}$ to $150 \mathrm{~nm})$ amorphous germanium films in multilayer structures from reflectance and transmission measurements. Multilayers are useful for a variety of technological devices and basic science investigations. There are several advantages of such structures for basic science studies. The addition of layer upon layer facilitates the study of phenomena where interfaces are important, such as intermixing, diffusion and where the mass of a single thin film might be inadequate for accurate measurements such as in the determination of thermal conductivity. ${ }^{-11}$.

In the present investigation the fact that in a multilayer the absorptance is a composite of the absorptances of many layers makes it possible to determine $n$ and $k$ of an ultrathin film germanium near the optical band gap. Furthermore, the effect of surface environment contamination is minimized.

Multilayers have proven to be objects worth studying in their own right. In single crystal epitaxy multilayers, so called superlattice structures, the existence of quantum size effects is now well established and a number of very interesting electronic and optoelectronic phenomenon have been studied. Since the advent of amorphous tetrahedral semiconductor alloys such as a-Si:H, which can possess noteworthy optoelectronic properties and a low density of states in the gap, multilayers containing amorphous materials have been prepared and characterized. 1 Among the various properties reported, one of the most interesting is a blue shift of the apparent band gap of a-Si:H/a-SiN $: H$ multilayers. 
A key property of crystalline superlattices which may be shared by their amorphous analogues is a shift to higher energy of the band gap of the material possessing the narrower band gap. In the case of crystalline multilayers, confinement of the carriers in an ultrathin, low band gap region sandwiched between wider band gap material has the quantum mechanical effect of increasing the optical band gap (a "blue" shift) of the narrow band material. The Kronig-Penny model is often used to explain the effect. Quantum confinement has also been used to
explain the reported increase in optical gap of a$\mathrm{Si}: \mathrm{H} / \mathrm{a}-\mathrm{SiN}_{\mathbf{x}}: \mathrm{H}$ multilayers.

There are other possibilities, such as the potential of structural and compositional differences between ultrathin and thicker thin film material. Cross contamination between layers is also a possibility. The incorporation of nitrogen from the a-SiN
posit into the a-Si:H layers will produce a material with higher band gap. There is also evidence that there could be excess hydrogen at the interfaces of layers.

The formal parallels between amorphous and crystalline multilayers are not sufficient to establish quantum confinement as the source of the blue shift in amorphous multilayers. Phase coherence of the carrier wavefunctions over the dimensions of the well to carrier wavefunctions over the dimensions of the well to increase the relevance of the results to parallel ex-
is often regarded as a requirement to see quantum perimental studies. The optical constants, over the size effects in multilayers. Amorphous materials lack required wavelength range, of the materials needed long range order. The optical absorption proper- to generate the synthetic reflectance and transmitties of a-Si alloys differ from crystalline silicon near tance traces had previously been determined from the band edge. Many of these differences are due individual ion beam sputtered a-SiN $\mathrm{x}_{\mathbf{x}}$ and $\mathrm{a}-\mathrm{Ge}$ thin to changes in transition probabilities and wavefunc- films.

tions arising from the loss of the long range order of crystalline silicon. Can the carriers maintain suffcient phase information in such a disordered matrix for quantum size effects?

The synthetic reflectance and transmittance traces were then analyzed by various coherent and incoherent effective medium theoretical approaches to extract the apparent band gap of the semiconduc-

It is also possible that part or all of the blue shift tor in the multilayer. Since the actual band gap of is an artifact of optical analysis. In most studies, the the semiconductor was known, the accuracy of each band gap has been extracted from transmission data method or approach could be evaluated by deterusing one or another of incoherent effective media mining the apparent band gap for specific multilayer approaches. Recently a few authors have addressed configurations.

the validity of such approaches. The issue, however, is not closed. The effect on band gap determination due to the presence of the dispersion in the refractive index of the dielectric layer have not been properly addressed in the past. In this study it will be shown to be potentially quite important. The determination of the structures and prop-by Taus. 12 This form of the Tauc plot is the form
erties, particularly the optical properties, of mate- commonly used by physicists in obtaining band gaps rials in ultrathin layers in multilayers is a rather from thin film reflection and transmission data. The formidable undertaking which must be approached intercept at the $x$ axis is defined as the optical band on several levels. A natural hierarchy is suggested: gap. first, the development of optical modeling and anal- Figure 1 contains data for both a-Ge and a-SiN . $_{\text {. }}$.
ysis tools, specifically determination of the appropri- The points plotted in Fig. 1 are calculated from a ate modeling and analysis techniques for computing realistic parametric model of the optical properties the optical constants from measured data; second, of ion beam sputtered a-Ge and a-SiN . development of preparation techniques and, structural characterization methods of ultrathin films;
The term "heterobases" can be applied to a- 
Ge/a-SiN multilayers. In contrast to homobasis multilayers like a-Si alloy/a-SiN $\mathrm{x}: \mathrm{H}$, in $\mathrm{a}-\mathrm{Ge} / \mathrm{a}-\mathrm{SiN} \mathrm{x}$ multilayers the network in the dielectric and semiconductor layers are based on different elements. The semiconductor layer is a-Ge, whereas the dielectric layer is based on silicon. The structural characterization of such multilayers is facilitated by the fact that the silicon and germenium continuing species are deposited in separate, adjacent layers. Using Raman spectroscopy to determine the number of adjacent, bonded silicon-germanium atoms, it is relatively easy to measure the amount of intermixing between layers. This aids in determining the extent to which intermixing between layers might be the cause for blue shifts in rnultilayers when such a shift is observed. The preparation and structural characterization of these ion beam sputtered multilayers is discussed elsewhere. ${ }^{13}$

The band gap of a-SiN $\mathrm{S}_{\mathrm{X}}$ depends on details of preparation. The a-SiN $\mathrm{x}$ dielectric produced by ion beam sputtering was found to be nitrogen deficient with the effect that the material is weakly absorbing above $1.8 \mathrm{eV}$. This is clear in Fig. 1. This produces absorption which adds to that of a-Ge. This absorption is, however, orders of magnitude less than the absorption of a-Ge even in the films with are mostly $\operatorname{SiN}_{\mathrm{x}}$.

The realistic parametric model used in generating the synthetic spectra of the present work is rooted in the modern understanding of the opti- cal properties of amorphous tetrahedral semiconductors. The absorptance, at moderate to high values of the absorptance, is described using the Tauc law; at low values of the absorptance an Urback Tail is used. The dispersion in the index is modeled as a power series in $\lambda^{-2}$, truncated after the $\lambda^{-6}$ term.

Further details can be found in Ref. 14. Note, as anticipated, that the graphical intercept matches in both cases the band gap used in the model to the expected accuracy of the procedure. The measured reflectance and transmittance of ion beam sputtered a-Ge and $\mathrm{a}-\mathrm{SiN}_{x}$ had been modeled using this parametric model in a previous study. ${ }^{13}$ The parameters obtained were used in the current study. Thus the $R$ and $T$ of the theoretical multilayer calculated in the present study mimic the optical properties of real multilayers containing a-Ge and $\mathrm{a}-\mathrm{SiN}_{\mathrm{x}}$ have important implications for the characterization of real multilayers discussed elsewhere. ${ }^{9}$

3. Computing theoretical reflectance and transmittance data

The $R$ and $T$ for a series of multilayers was computed. The multilayers consisted of 10 periods of a-Ge/a-SiN $x$ on a borosilicate glass substrate. The layer against glass was amorphous germanium while a-SiN ${ }_{x}$ was the layer facing out. The optical medium in front of the multilayer and behind the glass was assumed to have an index of 1.00 ; transmission re-

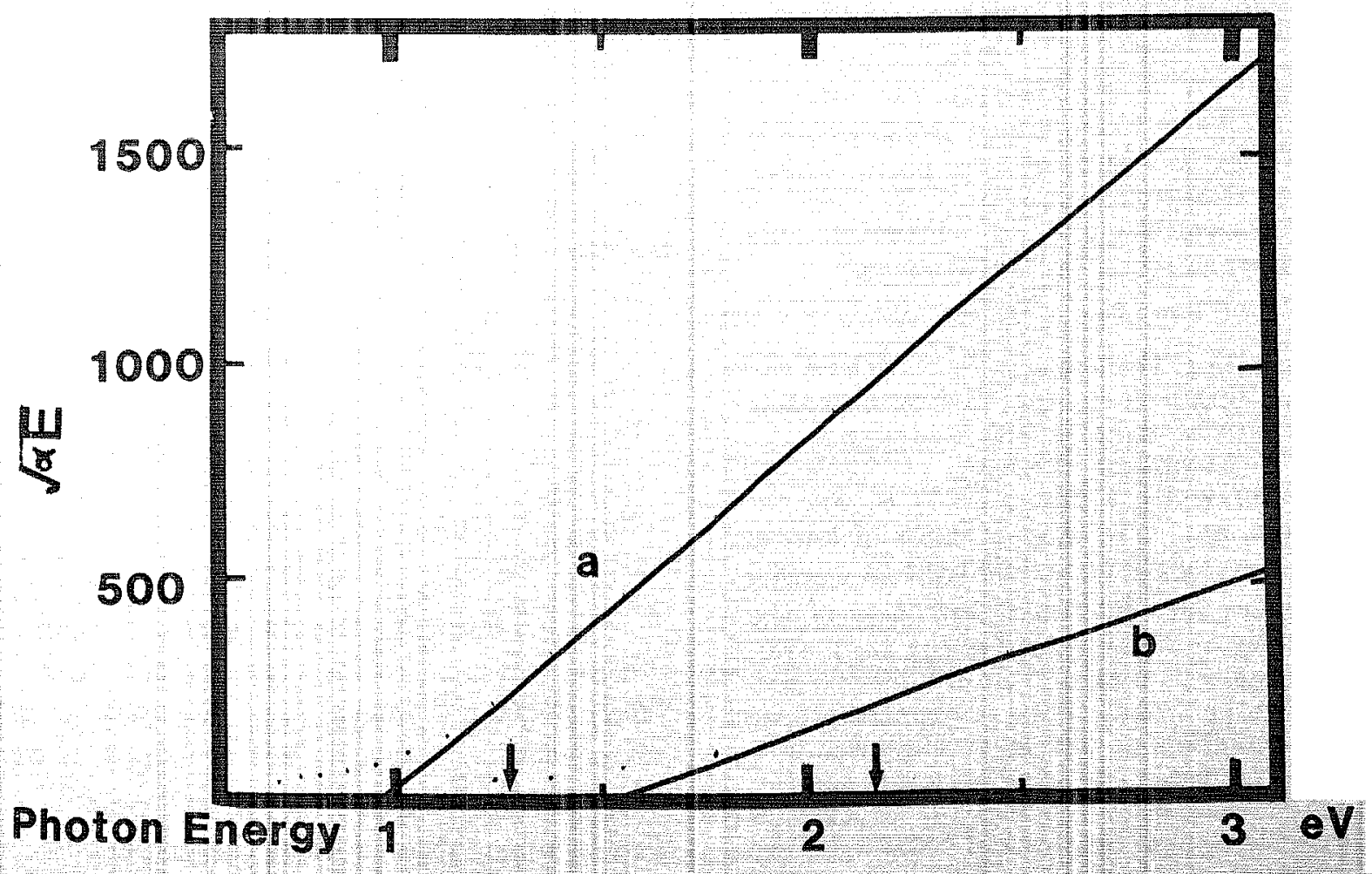

Fig. 1. Tauc Plot for values of a-Ge(curve a) and a-SiN (curve b) used in calculating synthetic multilayer reflectance $(R)$ and transmission $(T)$. Data is drawn from Ref, 13 , a study of ion beam sputtered a-Ge/a-SiNx multilayers: The ratch energy, where exponential tail is fitted on to the inner potion, is indicated with an arrow. 
flections between the substrates front and back surfaces were treated incoherently while the propagation of light in the multilayer was handled coher-

For one set of traces the thicknesses of the a-Ge

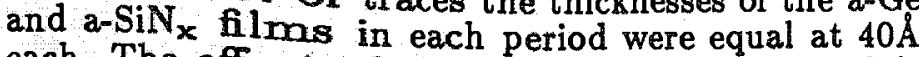
each. The effect of altering the a-Ge fraction while keeping the period thickness constant at $80 \AA$ was also investigated. In the most extreme case the $\mathrm{Ge}$ constituted only $10 \%$ of the stack.

It is appropriate to examine the assumptions made in deriving the commonly used form of the Tauc plot from the more fundamental $\sqrt{\epsilon_{2} E^{2}}$. Use the definitions

and

$$
\begin{aligned}
\epsilon_{2} & =2 n k \\
k & =\frac{\alpha \lambda}{4 \pi}
\end{aligned}
$$

$$
E \lambda=1.237 \mu \mathrm{m} \cdot \mathrm{eV}
$$

It can be obtained that

$$
\sqrt{\epsilon_{2} E^{2}}=\left(\frac{1.237 \mu \mathrm{m} \cdot \mathrm{eV}}{2 \pi} n \alpha E\right)^{1 / 2}
$$

Note that the simplification to $\sqrt{\alpha E}$ involves the approximation that the refractive index, $n$, is nondispersive, that is, that $n$ is a constant. This is found to be a sufficiently good assumption for many amorphous materials. It is rare then for physicists to take the extra effort to compute $n$ from the measured data and factor it in point by point in preparing Taus plots to compute $E_{g}$. Neglecting dispersion where it occurs can lead to difficulties in determining $E_{g}$. Nevertheless, its effect is usually ignored.

4. The Tauc Band gap of an Effective media

When the optical thickness (nd) of each of the layers in a multilayer is comparable to a quarter of the wavelength of light strong interference can be expected. The optical thickness of the layers in multilayers for which a blue shift in $E_{g}$ are observed, however, are more than a factor of twenty less than the wavelength of visible light $(\lambda>4000$ A). In such cases the electromagnetic field of the light varies slowly over one period of the multilayer. Thus interference effects arising from ultrathin layers are found to be small. Thus it appears proper to treat such materials as one layer composed of a single effective inaterial possessing optical properties intermediate between a-Ge and a-SiN ${ }_{x}$. Approaches for averaging the optical constant of composites are termed effective medium theories (EMT). These can be coherent or incoherent with respect to the effect of the flm on the substrate and the optical constants can be obtained point by point or as parameters for some functional form.

In fact the reflectance and transmittance spectra of the multilayer of this study, real or simulated, resemble those of a single semiconductor film on a substrate. Since the dielectric is transparent at the energy band edge of the sermiconductor it is relatively common for researchers to extract a band gap from the composite film and attribute it to the semiconductor without further qualification. It is the appropriateness of this assignment that is being investigated,

It can be shown that in the illumination of lamellar structures at normal incidence to the surface, effective medium approaches take a particularly simple form. The complex effective dielectric constant, $\tilde{\epsilon}_{f}$, is just the volume weighted average of $\tilde{\epsilon}$ for each of the layers.

Thus

$$
\tilde{\epsilon}_{f}=V_{\mathrm{Ge}} \tilde{\epsilon}_{\mathrm{Ge}}+\left(1-V_{\mathrm{Ge}}\right) \tilde{\epsilon}_{\mathrm{SiN}}
$$

remembering that

$$
\tilde{\epsilon} \equiv \epsilon_{1}+i \epsilon_{2}
$$

where

$$
\epsilon_{1}=n^{2}-k^{2}
$$

and

$$
\epsilon_{2}=-2 n k
$$

and, similarly,

$$
\tilde{\epsilon}_{f}=\epsilon_{1, f}+i \epsilon_{2, f}
$$

where $\tilde{\epsilon}_{f}$ is the effective complex dielectric constant and $\epsilon_{1, f}$ and $\varepsilon_{2, f}$ are the real and imaginary components respectively.

So

$$
\epsilon_{i f}=-V_{\mathrm{Ge}} \epsilon_{i, \mathrm{Ge}}+\left(1-V_{\mathrm{Ge}}\right) \epsilon_{i, \mathrm{SiN}}
$$

If

$$
\begin{gathered}
k_{\mathrm{SiN}}=0 \\
k_{f}=V_{\mathrm{Ge}} \frac{n_{\epsilon}}{n_{f}} k_{\mathrm{Ge}} \\
\sqrt{\alpha_{f} E}=\sqrt{V_{\mathrm{Ge}} n_{\mathrm{Ge}} / n_{f}} \sqrt{\alpha_{\mathrm{Ge}} E}
\end{gathered}
$$

Thus, if $\sqrt{\alpha_{\mathrm{Ge}} E}$ obeys Taus law then so will $\sqrt{\alpha_{f} E}$ provided $n_{G e}$ and $n_{f}$ are constants or vary in such a way that $n_{\mathrm{Ge}} / n_{f}$ remains a constant.

This argument thus provides the justification for computing the effective optical constants of the multilayer as though it were a single layer and assigning the Tauc band gap to a-Ge. The assumptions that 
index of refraction terms can be treated as constants is, however, highly suspect for accurate, as opposed to qualitative, determinations of the band gap.

\section{Results and Discusgion: $\mathbb{E M T}$ Models}

The mosi frequently used method of obtaining $T$ and $R($ or $\alpha)$ of $\alpha n$ amorphous semiconductor alloys is to model $R$ and $T$ incoherently by, that is, to ignore coherent thin film interference effects. Since band edge in the trantsmission spectrum of their films with a thickness $\geq 3000 \AA$, the common approach is to allow for multiple reflections but to neglect constructive/destructive interference in the calculation of the absorption constant.

We have used two such methods to calculate $\alpha$ from a 10 period $(40 \AA / 40 \AA)$ a- SiN $_{\mathbf{x}} /$ a-Ge multilayers. The first method uses $R$ and $T$ simultaneously to form the ratio $(1-R) / T$. Residual interference effects are partially eliminated in such a quantity. The quantity is fur her manipulated to give $\alpha$. The second method uses $R$ to calculate the effective index of the multilayer as a function of wavelength. From the transmission, the quantity $e^{-\alpha d}$ is found, which can be solved for $\alpha_{0}{ }^{15}$

Curves a and b in Fig. 2 are, respectively, plots of $\sqrt{\alpha E}$ vs. $E$ for $\alpha$ derived from each incoherent approach.
It can be seen that the apparent intercept is 1.06 $\mathrm{eV}$ in both cases. Thus the incoherent EMT approaches can produce an entirely artificial blue shift in the band gap. The effect is a relatively minor $7 \%$, however. We have found that this offset is relatively constant as the thickness of the layers goes to zero. In contrast, for actual a-Si:H/a-SiN $: H$ multilayers the thinner the layers the more pronounced is the blue shift. Therefore an optical analysis blue shift can be separated from a quantum well blue shift.

The fact that incoherent optical modeling can produce a blue shift, albeit relatively small, provides impetus to do coherent optical modeling. The same coherent fitting program that was used to determine from $R$ and $T$ the optical constants of amorphous Ge and amorphous $\mathrm{SiN}_{\mathrm{x}}$ in the a-Ge/a-SiN $\mathrm{S}_{\mathbf{x}}$ multilayer was used to compute the apparent $n$ and $k$ of the synthetic multilayer treating it as a single effective median thin film.

Figure 3, curve $a$, is a Tauc plot employing the apparent $\alpha$ of a $40 \AA / 40 \AA$ multilayer where $n$ and $k$ were calculated coherently. In contrast with the a-Ge spectra (see Fig. 1) which y ields, by construction, a straight line (above the Urbach tail) on the Tauc plot, the graph of $\sqrt{\alpha E}$ above the tail is not a straight line. The line curves upward at high energy. The effect is not significant when values just above the tail region are used. The apparent band gap is

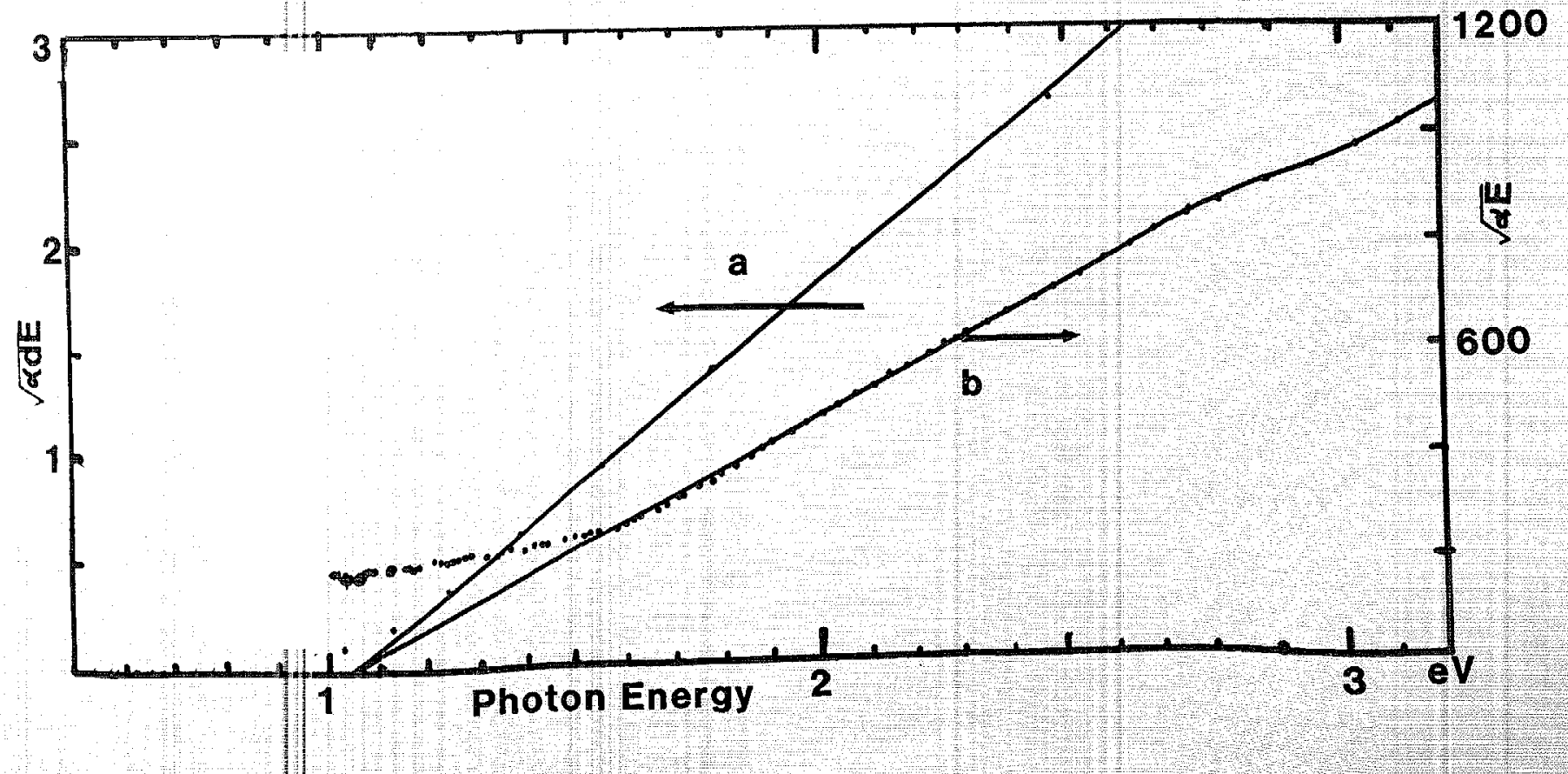

Fig. 2. Taus Plot ob thined from synthetic multilayer reflectance and transmission traces using incoherent effective media approthes. The multilayer was 10 periods of $(40 \AA-a-G e) /\left(40 \AA-\mathrm{SiN}_{x}\right)$ on glass backed by air.

a. Procedure d to Canella and Burdick based on analysing the quantity, $(1-T) / R$.

b. Procedure d, to Allred and Walter, based on Janai $e t a l,{ }^{15} n$ of the film is obtained from $R$ and $\alpha$ is obtained fro

$T$ and the $n$, of the film, air and the substrate. 
about $1.01 \mathrm{eV}$, just $2 \%$ higher than the actual $E_{g}$. When the highest energy points are used in the extrapolation of the band gap, however, the apparent band gap is $1.12 \mathrm{eV}$, approximately $13 \%$ higher than the actual band gap.

The source of the discrepancy is the curvature of the graph. That this is due to dispersion is made evident in curve b (Fig. 3) which uses the synthetic $R$ and $T$ spectra of a $90 \% \mathrm{SiN}_{\mathrm{x}} / 10 \%$ a-Ge multilayer to calculate an EMT $\alpha$, and $n$. Here the curvature of $\sqrt{\alpha E}$ is sufficient to make it difficult to define what is the region above the tail and to throw the extrapo-

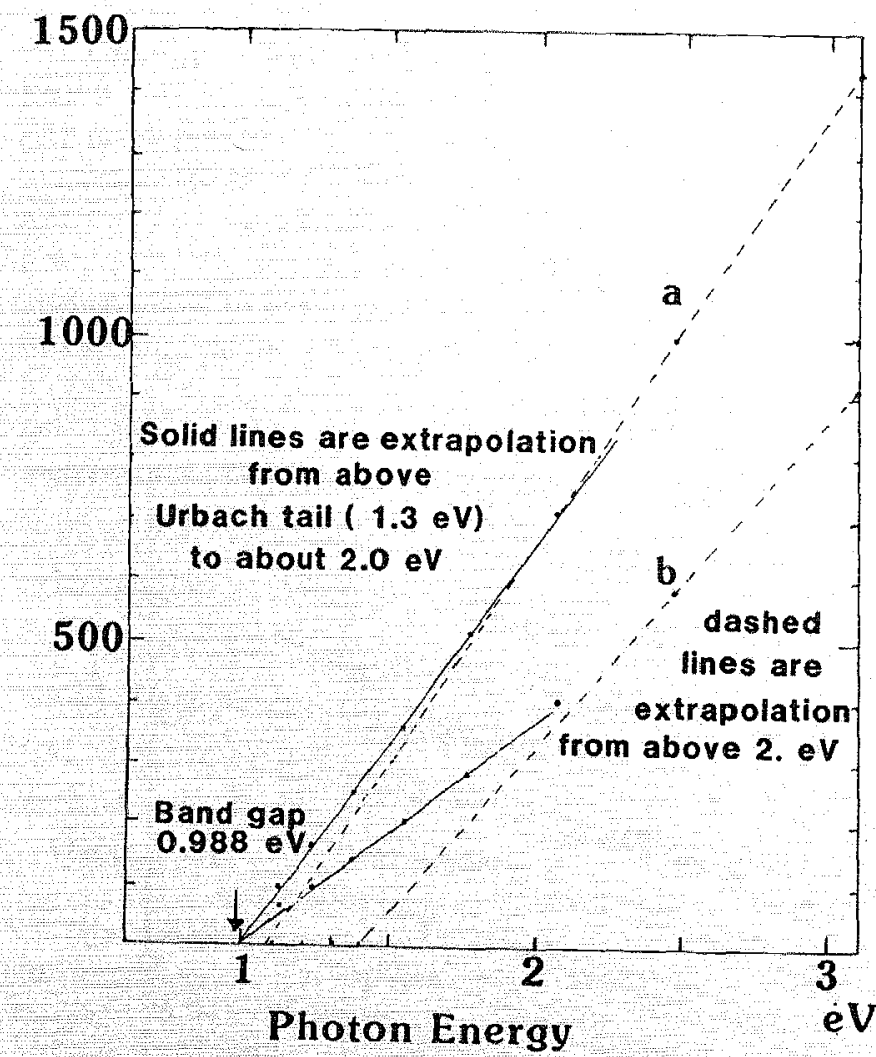

Fig. 3. Tauc Plot for values of $\alpha$ obtained with a coherent effective media approach for synthetic a$\mathrm{Ge} / \mathrm{a}-\mathrm{SiN}_{x}$ multilayers

a. 10 periods of $40 \AA / 40 \AA$ a-Ge/a-SiN on glass.

b. 10 periods of $4 \AA / 36 \AA$ a-Ge $/ a-S_{1}$ on glass.

The presence of a-SiN $x$ increases the value of $\sqrt{a E}$ at higher photon energies. lated band gap as high as $1.37 \mathrm{eV}$ when high energy data is used. A rey point is that if data in the 1.5 to 2.5 energy range is used, which, for a-Ge, is the practical range to choose, the estimated band gap is $1.1 \mathrm{eV}$, only $12 \%$ higher than the actual band gap. It is worth asking how such a nonlinear graph arises fiom a multilayer which contains as the most absorbing material a-Ge when the plot of $\sqrt{\alpha_{G e} E}$ vs. $E$ itself is a straight line. Whereas $\mathrm{SiN}_{\mathrm{x}}$ is largely transparent in the region of interest it infuences the apparent band gap of the composite through its index of refraction which distorts the $\sqrt{\alpha E}$ form of the Tauç plot.

The effect of dispersion need not be a serious complications for most quantum well studies. Multilayers which are $90 \%$ dielectric (a-SiN $x$, for example) and only $10 \%$ semiconductor (a-Ge or a-Si:H) represent extreme cases. In most films where evidences of quantum confinement effects have been claimed, the relative partitioning was closer to 50:50. While dispersion in the index of the dielectric component even for 50:50 multilayers does have an influence on the apparent band gap which must not be ignored for quantitative comparisons, the effect is smaller than the shifts in band gap in many cases. It is, therefore, not possible to discount all of the blue shift observed to optical analysis effects.

On the other hand, optical analysis effects must not be ignored. While incoherent models might still be favored for qualitative work because the calculations require minimal computer time and no iterations, the extent to which incoherent models consistently missed the band gap is significant. Coherent modeling is required for accurate work, on the other hand, full coherent optical modeling of the multilayer taking into account all of the layers is not desireable either. Such an approach can yield the correct band gap, but is intolerably time consuming and computationally demanding. Convergence is frequently slow and false minima are a possibility. It appears that coherent effective medium optical modeling approach of the type shown is Fig. 3 are a viable alternative. The time taken in setting up the problem and abstracting band gaps is relatively modest even for desk top computers. False minima are generally not a problem. In addition, coherent EMT calculates band gaps which generally differ from the actual band gap by only a few percent even when dispersion is significant.

We have observed further that this error can be decreased even further by explicitly taking into account the effects due to index of refraction. This can be done by using the $E \sqrt{\epsilon_{2}}$ version of the Taus law or forming the ratio $n_{\mathrm{Ge}} / n_{f}$ at all energies. Since the values of $n_{\mathrm{Ge}}(\lambda)$ are generally unknown for films in multilayers, in calculating the ratio the values of a-Ge from conventional thin films can be used. 


\section{Summary}

Using the measured optical constants of a.Ge and a-SiN ${ }_{x}$ in multilayers we have generated synthetic $R$

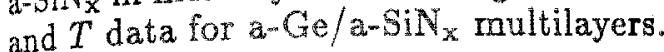

These synthetic traces were analyzed by a number of commonly used effective media theory ( $E M T$ ) procedures to obtain the band gap of the multilayer. of the various approaches for obtaining band gaps which were studied coherent $E M T$ is particularly ncteworthy. Whereas both incoherent effective media approaches yield a band gap about $8 \%$ high, the coherent approach determined the band gap to within $2-3 \%$ of the actual value $(1.02$ versus 0.986 eV). The coherent $E M T$ approach requires computer iteration but the procedure is compatible with desk top computers and is far less time consuming than a complete coherent model which dealt with each layer in the stalk.

Refractive index dispersion can have a large effect on the determination of the band gap in cases containing much less absorber than dielectrics. The importance of this effect had not been previously appreciated for such problems. Versions of the Tauc plot which explicitly account for dispersion $\sqrt{\epsilon_{2} E^{2}}$ should be employed when dispersion effects are large. It appears that artifacts in optical analysis can account for some, but not all, of the blue shift in $E_{g}$ seen in amorphous multilayers. Nevertheless, data supporting a "blue shift" in a-Si:H multilayers could be reexamined in light of this study.

\section{Acknowledgements}

We wish to acknowledge the support of Energy Conversion Devices, Inc. during the computational portion of this work. D.D. Allred thanks BYU and the Center for $X$-Ray Optics for subsequent support and encouragement. We thank Joseph Burdick and Lee Walter for incoherent modeling and Rea Newell and other members of the BYU Department of Physics and Astronomy staff for assistance in preparing the manuscript. This work is dedicated to Dwight Gibson (dec.) whose enthusiasm at ECD inspired us.

\section{References}

1. B. Abeles, and T. Tiedje, “Amorphous semiconductor superlattices," Phys. Rev. Lett, 51, 2003$6(1983)$.

2. P. Hollingsworth-Smith, and H.S. Gurev, "Silicon dioxide as a high temperature stabilizer for silver films," Thin Solid Fims, 45, 159 (1977).

3. D.E. Aspnes in Handbook of Optical Constants in Solids, Edward D. Palik, ed, Academic Press, Orlando, pp. 89-110 (1985).
4. J. Pilion, K. Vedam, J.E. Yehoda and R. Messier, "Thickness dependence of optical gap and void fraction for sputtered amorphous germanium," Phys. Rev. B, 35, 9368, 1987.

5. D.Y. Smith, in Handbook of Optical Constants in Solids, Edward D. Palik, ed., Academic Press, Orlando, pp. 374-376 (1985).

6. J.M. Bennett, App. Opt., 15, 2765 (1976).

7. A.L. Greer and F. Spaepen "Diffusion" in Synthetic Modulated Structures, L.L. Chang and B.C. Giessen, eds., Academic Press, Inc. Orlando, pp. 419-484 (1985).

8. D.D. Allred, J. Gonzales-Hernandez, O.V. Nguyen, D. Martin and D. Pawlik, "Raman scattering and $x$-ray diffraction characterization of amorphous semiconductor multilayer interfaces," J. Mat. Res., 1, 468 (1986).

9. J. Gonzalez-Hernandez, D.D. Allred, and O.V. Nguyen, "Anneal Induced Changes in Amorphous Semiconductor Multilayers," in John D. Dow and Ivan K. Schuller, eds, Interfaces, Superlattices and Thin Films, Proc. Material Research Society 77, Pittsburgh, PA, pp. 665-670 (1987).

10. K.L. Chopra, and Prem Nath "Thermal conductivity of ultrathin metal films in multilayer structures," J. of Appl. Phys., 45(4), 1923 (1974).

11. Prem Nath and K.L. Chopra, "Thermal conductivity of amorphous and crystalline germanium and GeTe," Phys. Rev. B, 10(8), 3412 (1974).

12. J. Tauç, R. Grigorovici and A. Vancu, Phys. status solidi, 15, 627 (1966).

13. D.D. Allred, J. Gonzalez-Hernandez, "Optical and structural properties of a-Ge/a-SiN multilayers," to be published.

14. Robert E. Edgerton and David Short, in "Optical characterization of thin semiconducting thin films on transparent substrates," Basic Properties of Optical Materials (OM85), Proc. of the NBS Conf. on, Dept of Commerce, Gaithersburg MD. (1985).

15. M. Janai, D.D. Allred, D.C. Booth and B.O. Seraphin, "Optical properties and structure of amorphous silicon films prepared by CVD, Solar Energy Materials, 1, 11 (1979). 